\title{
Can Bars be Destroyed by Central Mass Concentrations?
}

\author{
E. Athanassoula \\ Observatoire de Marseille, 2 place Le Verrier, 13248 Marseille cedex 04 , \\ France \\ W. Dehnen \\ Department of Physics 89 Astronomy, University of Leicester, Leicester \\ LE1 7RH, UK \\ J. C. Lambert \\ Observatoire de Marseille, 2 place Le Verrier, 13248 Marseille cedex 04, \\ France
}

\begin{abstract}
We use $N$-body simulations to follow the evolution of bars in the presence of a central mass concentration (CMC). We find that the bar amplitude decreases in response to the CMC, but the bar is not totally destroyed unless the CMC mass is several percent of that of the disc. More centrally concentrated CMCs are more efficient. The bar evolution does not stop after the CMC has fully grown, but continues well after that time.
\end{abstract}

\section{Introduction}

Both strong bars and CMCs can be found in a large fraction of disc galaxies. Examples of the latter can be black holes or central concentrations of molecular gas. It is thus necessary to study the dynamics of cases where both bars and CMCs are present in the same disc. Initially this was attempted by studying the orbits of individual particles in rigid potentials which have both a CMC and a bar component (Hasan \& Norman 1990, Hasan, Pfenniger \& Norman 1993). Such studies aim towards an understanding of the stability of the individual periodic orbits, which is necessary in order to assess the possible building blocks from which a bar can be built. They can not, however, give quantitative information on how a bar will evolve in the presence of a black hole. Thus these works were followed by $N$-body simulations, either 2D (Norman, Sellwood \& Hasan 1996, Hozumi \& Hernquist 1998), or 3D but with mainly rigid haloes (Norman et al 1996 , Shen \& Sellwood 2003). Here we will present results of $N$-body simulations in which both the disc and the halo are live, i.e. composed of particles.

\section{Results}

The initial conditions for our simulations were taken from one of the simulations of Athanassoula (2003). These started with an exponential disc of unit mass and scale length. This sets the units of mass and length. For the model described 
here we chose a halo with a core radius half the disc scale length and a mass five times that of the disc. A bar grows spontaneously in this simulation and evolves by emitting angular momentum, which is absorbed by the outer disc and the halo (Athanassoula 2003). For the simulations we present here we use as initial conditions a time at which the bar has grown quite strong. At that time we introduce gradually a CMC, which for simplicity is modeled by a Plummer sphere. We have tried a number of growth times $\left(T_{\text {grow }}\right)$, of final CMC masses $\left(M_{C M C}\right)$, and of CMC scale lengths $\left(R_{C M C}\right)$. In this section we will discuss some results obtained with $T_{\text {grow }}=100, R_{C M C}=0.01$ and $M_{C M C}=0.1$ or 0.05 , all measured in computer units. Results for other parameter values will be very briefly mentioned in the next section and a complete account of our results will be given elsewhere. The evolution was followed with a treecode (Dehnen 2000, 2002).
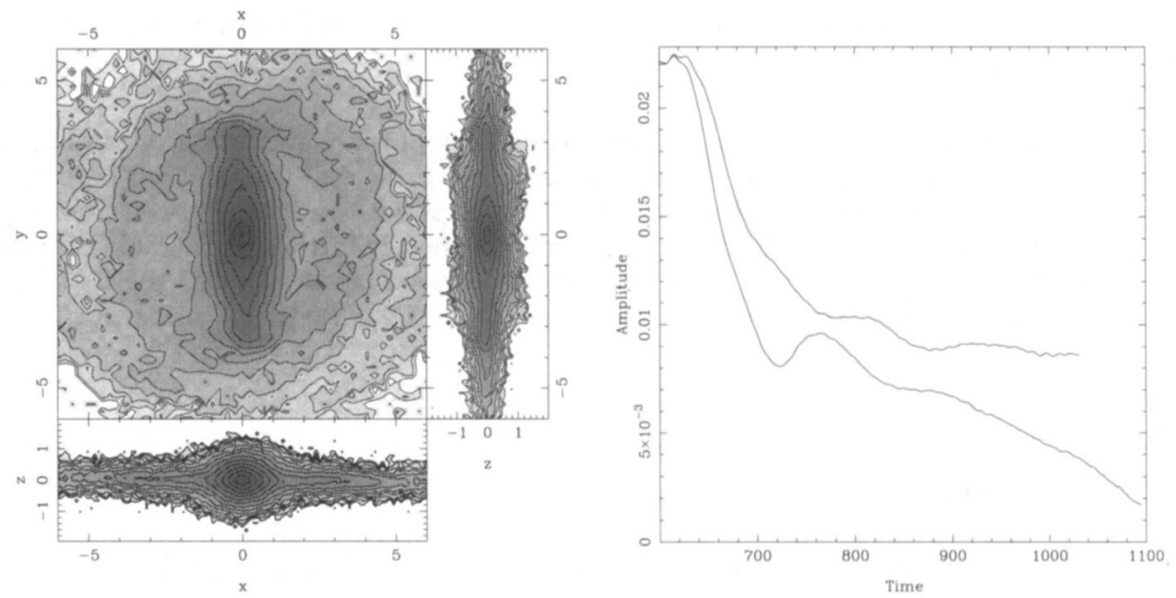

Figure 1. The left panel shows the three orthogonal views of the disc component at the time the CMC is introduced. The right panel shows the bar strength for the two simulations discussed in section 2 , as a function of time.

The left panel of Fig. 1 shows the three orthogonal views of the disc component at the time the CMC is introduced. Seen face-on, the bar is reminiscent of the strong bars observed in real galaxies. It is long and thin and its isodensities have a rectangular-like shape in the outer parts (Athanassoula \& Misiriotis 2002). Seen edge-on and side-on, i.e. with the line of sight along the bar minor axis, it displays a characteristic peanut shape.

Fig. 2 shows the disc component 440 computer time units after the $\mathrm{CMC}$ has been initially introduced. Note that in the calibration introduced by Athanassoula (2003) the unit of time is $1.4 \times 10^{7} \mathrm{yrs}$, so that 440 computer units correspond roughly to 6 Gyrs. In the simulation with $M_{C M C}=0.05$ the bar is still present, but is considerably shorter and less thin. Its isodensities are more elliptical-like and in the innermost parts they are near-circular. Seen side-on, it is now boxy. For $M_{C M C}=0.1$ the bar has nearly disappeared (indeed it does so even more at later times of that simulation) and seen edge-on it has an oblate shape. 

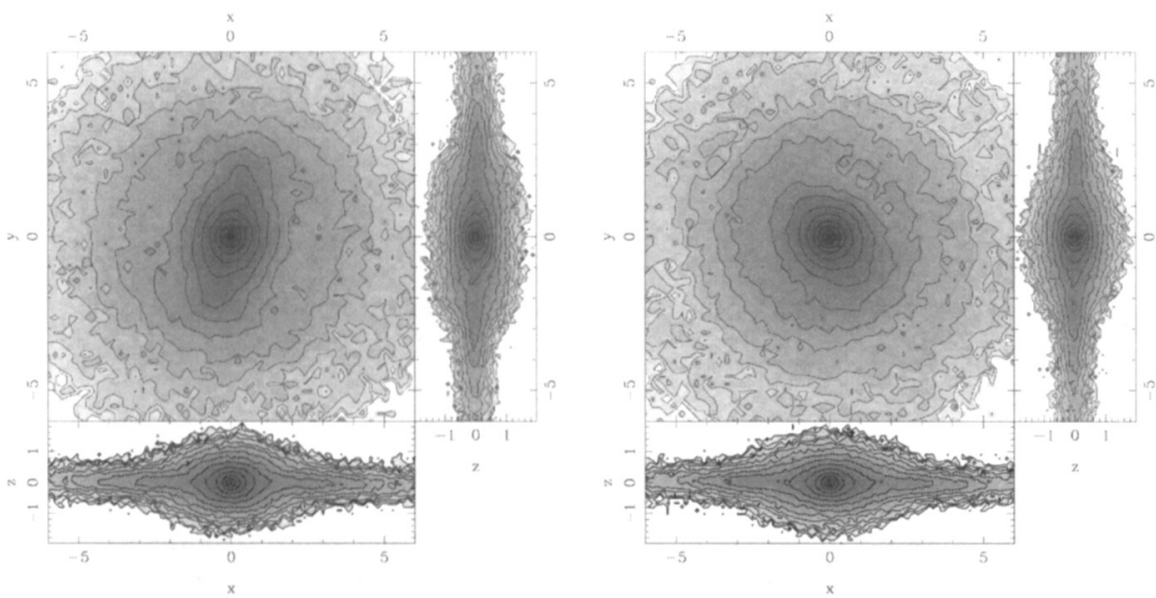

Figure 2. Three orthogonal views of the disc component towards the end of the simulations. The left panel corresponds to the simulation with $M_{C M C}$ $=0.05$ and the right one to the simulation with $M_{C M C}=0.1$.

The strength of the two bars (here simply defined as the maximum of the $m=2$ Fourier component of the density in arbitrary units) is compared as a function of time in the left panel of Fig. 1. The time is given in computer units. We note that in the first 20 to 30 time units the bar strength stays roughly constant. This is followed by a phase of sharp decrease, which lasts roughly 100 time units. This is followed by a third phase, where the bar still decreases, but now considerably less fast. This plot, and many similar ones for other simulations, shows clearly that the decrease of the bar amplitude does not stop after the CMC has reached its maximum mass, but continues well after that time.

Fig. 3 shows the amplitude of the $m=2$ component of the density as a function of radius and of time. At the time when the CMC is introduced the $m=2$ component is small in the innermost regions and grows outwards to reach a maximum, and then decreases. At later times the amplitude of the $m$ $=2$ decreases. This is achieved from the centermost and the outermost parts simultaneously. This is consistent with what was seen in Fig. 2, which showed that the innermost contours become rounder and the length of the bar decreases considerably. All this is much clearer for the case with $M_{C M C}=0.1$, but can also be seen for $M_{C M C}=0.05$. Similar plots (not shown here) for the $m=4$ component show that this component decays much faster than the $m=2$ one. As a result, the isodensities become elliptical-like before the bar disappears.

\section{Summary and Discussion}

The main results from a number of simulations (not shown here) are that :

- CMCs with larger masses and/or smaller scale lengths are more efficient in destroying the bar. 

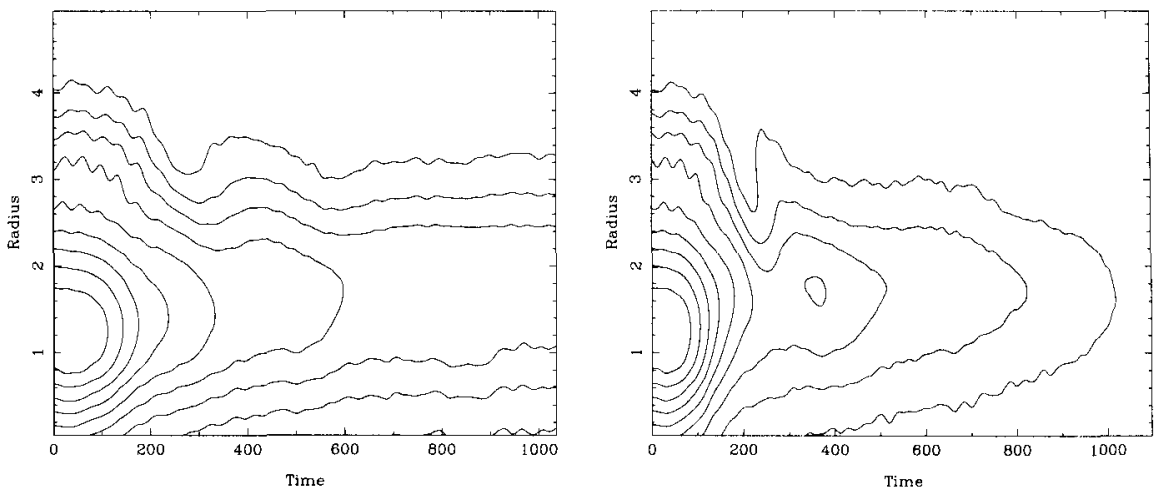

Figure 3. Amplitude of the $m=2$ component of the density as a function of radius and of time. The left panel corresponds to the simulation with $M_{C M C}$ $=0.05$ and the right one to the simulation with $M_{C M C}=0.1$.

- The mass and the scale length of the CMC necessary to destroy the bar depend on the bar model.

- For all models we tried, a CMC mass of at least several percent of the disc mass is necessary in order to destroy the bar.

- The final result does not depend much on the CMC growth time

- The evolution does not stop after the CMC has reached its maximum mass.

These and further results will be discussed further elsewhere, where we will present also comparisons with previous analysis and with observations.

\section{References}

Athanassoula E. 2003, MNRAS, 341, 1179

Athanassoula E., \& Misiriotis, A. 2002, MNRAS, 330, 35

Dehnen, W. 2000, ApJ, 536, L39

Dehnen, W. 2002, J. Comp. Phys., 179, 27

Hasan, H., \& Norman, C. 1990, ApJ, 361, 69

Hasan, H., Pfenniger, D., \& Norman, C. 1993, ApJ, 409, 91

Hozumi, S., \& Hernquist, L. 1998, astro-ph 9806002

Norman, C., Sellwood, J. A., \& Hasan, H. 1996, ApJ, 462, 114

Shen, J., \& Sellwood, J. A. 2003, AAS Meeting 203, 91, 6 (astro-ph 0303130) 